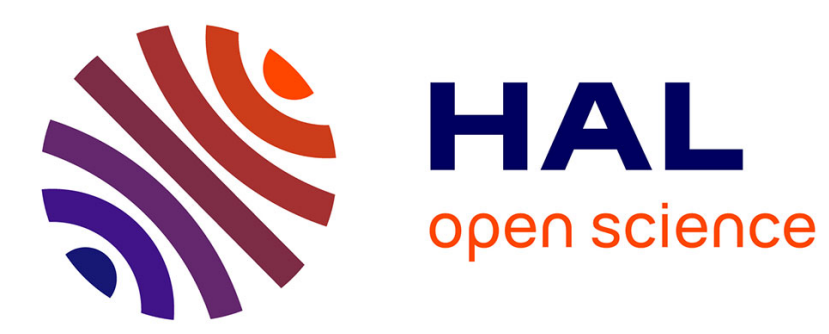

\title{
POLARIZATION AS A TOOL FOR REVEALING SUPERSYMMETRIC PARTICLES
}

\author{
F. Renard
}

\section{To cite this version:}

F. Renard. POLARIZATION AS A TOOL FOR REVEALING SUPERSYMMETRIC PARTICLES. Journal de Physique Colloques, 1985, 46 (C2), pp.C2-23-C2-30. 10.1051/jphyscol:1985203 . jpa00224512

\section{HAL Id: jpa-00224512 https://hal.science/jpa-00224512}

Submitted on 1 Jan 1985

HAL is a multi-disciplinary open access archive for the deposit and dissemination of scientific research documents, whether they are published or not. The documents may come from teaching and research institutions in France or abroad, or from public or private research centers.
L'archive ouverte pluridisciplinaire HAL, est destinée au dépôt et à la diffusion de documents scientifiques de niveau recherche, publiés ou non, émanant des établissements d'enseignement et de recherche français ou étrangers, des laboratoires publics ou privés. 


\title{
POLARIZATION AS A TOOL FOR REVEALING SUPERSYMMETRIC PARTICLES
}

\author{
F.M. Renard
}

Département de Physique Mathématique`, Université des Sciences et Techniques du Languedoc, 34060 Montpeziier Cedex, France.

Resumé - Les particules supersymétriques diffèrent des particules ordinaires par une demi-unité de spin. Leur production dans les collisions de faisceaux polarisés présente donc des asymétries particulières qui devraient permettre de les identifier plus facilement. Des exemples typiques sont la production de gluinos dans les collisions proton-antiproton, de photons directs à grand moment transverse, de fermions scalaires dans les collisions $e^{+} e^{-}$. Nous présentons les premiers résultats d'un travail en cours sur la production de particules supersymétriques dans les collisions $e^{+} e^{-}$polarisées.

Abstract - Supersymmetric particles differ from ordinary ones by half a unit of spin. This lead to special asymmetries in their production with polarized beams which should allow a better identification. Typical examples are gluino production in $p \bar{p}$ collisions, direct photon production at large $p_{1}$ and scalar fermion production in $e^{+} e^{-}$collisions. We report on a work in progress about supersymmetric particle production in polarized $e^{+} e^{-}$collisions.

\section{I - INTRODUCTION}

The idea of Supersymmetry (fermion-boson symmetry) is at least 13 years old $/ 1 /$. Applied to quantum field theory it leads to very interesting possibilities like extension of space-time symmetries by internal symmetries, reduction of divergences in perturbation theory, inclusion of gravity $/ 2 /$. Phenomenological models however appeared difficult to construct $/ 3 /$. Ordinary particles do not fall into supersymmetric multiplets unless one assumes that they are associated with new particles (the Susy-partners) differing by half-a-unit of spin. Examples are $\left(\tilde{l}_{L}, \tilde{l}_{\mathrm{R}} ; \boldsymbol{l}\right)$, $\left(\tilde{q}_{L}, \widetilde{q}_{R} ; q\right),\left(H^{ \pm} ; \widetilde{W}_{1}, \widetilde{W}_{2} ; w^{ \pm}\right),\left(H^{0} ; \tilde{Z}_{1}, \widetilde{Z}_{2} ; z^{0}\right),(\tilde{r} ; \gamma),(\tilde{g} ; g)$. One can check that in each supermultiplet the number of fermionic degrees of freedom is equal to the number of bosonic ones. $\tilde{\boldsymbol{l}}_{I_{L}, \mathrm{R}}$ and $\tilde{q}_{L, R}$ are spin zero particles (scalar leptons and quarks associated to the left or right chiral leptons and quarks) $; \mathrm{H}^{ \pm}$and $\mathrm{H}^{\circ}$ are charged and neutral scalar bosons (Higgses); $\widetilde{W}_{1}$ and $\widetilde{W}_{2}$ are Dirac fermions (Winos); $\widetilde{Z}_{1}, \widetilde{Z}_{2}, \widetilde{Y}$ and $\widetilde{g}$ are two-component majorana fermions (Zinos, photino, gluinos). As none of them have yet been experimentally observed this means that susy is broken in the particle spectrum, i.e. the partners have much higher masses.

In spite of that Susy got an impressive theoretical interest during the last ten years and which keeps growing $/ 3,4 /$. The main reasons are that it gives a possibility of constraining the scalar sector of the electroweak theory (Higgs mechanism). The Fermi scale ( $W$ mass) can be found as a consequence of Susy breaking. Susy may solve the hierarchy problem in Grand Unified Theories. Realized locally supersymmetric invariance describes gravity and gives a hope of establishing a unique

\footnotetext{
^Physique Mathématique et Thẻorique, Equipe de Recherche Associêe au CNRS
} 
description of all particles and interactions.

Various models of Susy breaking have been proposed /3,4/. Most of them lead to the minimal spectrum represented in Fig. 1 . A new quantum number ( $R$ ) is also introduced $(\mathrm{R}=0$ for usual particles and \pm 1 for the Susy-partners). This constrains Susy particles to be produced in pairs and to decay by cascade down to the lightest stable one (generally the photino $\widetilde{\gamma}$, which could eventually decay very weakly into a photon and a gravitino). Examples : $\tilde{\boldsymbol{l}} \rightarrow \boldsymbol{l}+\tilde{\gamma} ; \tilde{q} \rightarrow q+\tilde{g} ; g \rightarrow g+\tilde{\gamma}$ or $q \bar{q}+\tilde{\gamma}$;

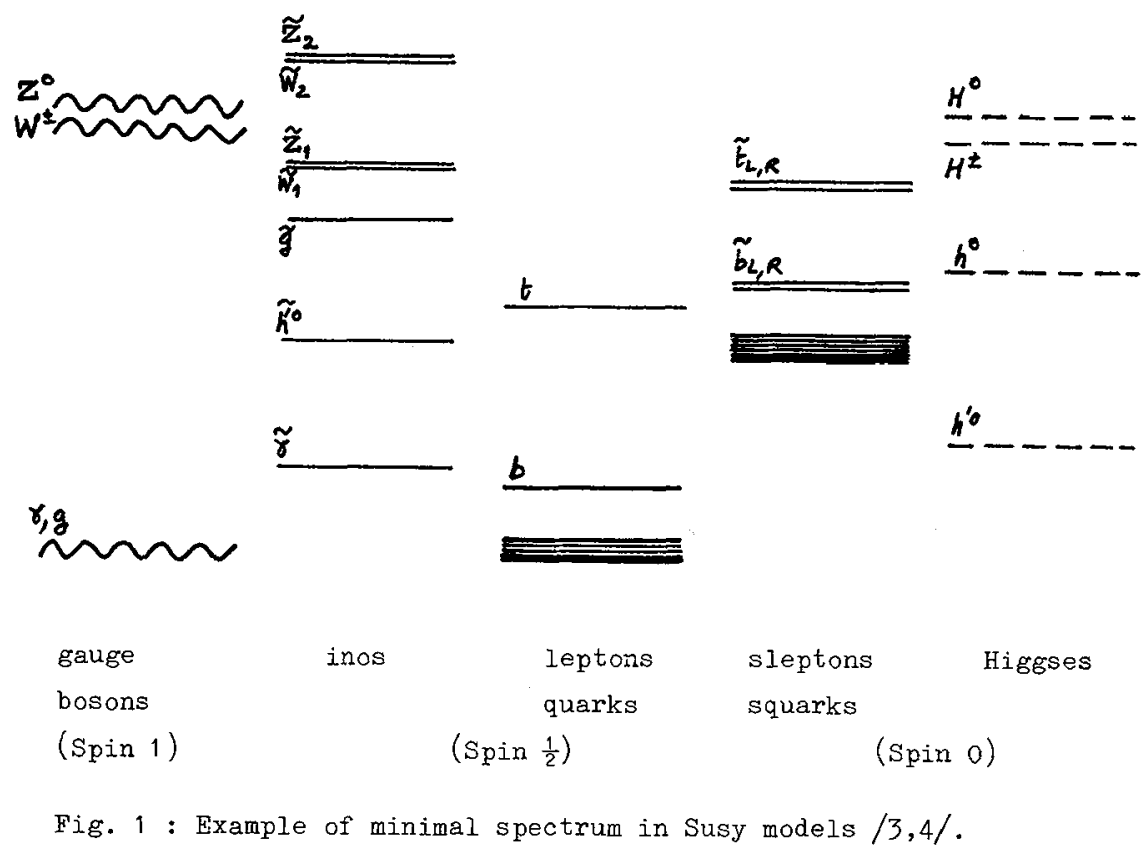

WI, $\widetilde{Z} \rightarrow(\boldsymbol{l} \boldsymbol{l}$ or $q \bar{q})+(\tilde{\gamma}$ or $\tilde{g}) ; \ldots$ etc. This lightest particle will escape detection so the production of Susy particles is always characterized by missing energymomentum.

Negative experimental searches have been carried in $e^{+} e^{-}$and in hadronic collisions /5,6/. From the absence of $\mathrm{e}^{+} \mathrm{e}^{-} \overrightarrow{\tilde{l}_{\boldsymbol{l}}}$ and $\tilde{\mathrm{qq}}$ at Pep and Petra one can set $\operatorname{man}_{\boldsymbol{\ell}, \tilde{q}}^{\sim} \gtrsim 16 \mathrm{GeV}$. At Pep the reaction $\mathrm{e}^{+} \mathrm{e}^{-}-\tilde{e e \gamma}$ has been especially studied in order to push the limit on the selectron mass slightly higher $\left(m_{\overparen{Z}} \gtrsim 23 \mathrm{GeV}\right)$. The reaction $e^{+} e^{-}-\widetilde{\gamma} \widetilde{r}$ is even sensitive to higher values through the influence of the selectron propagator but its analysis requires a good control of the background /6/. Beam dump experiments gave the limit $\mathrm{m} \approx 3 \mathrm{GeV}$. Various tests have been proposed / $/$ for $p\left({ }_{p}\right)$ colliders in particular in connection with $W$ and $Z$ production (decays of $W, Z$ in pairs of Susy particles or anomalous $W, Z$ production due to Susy processes). The recent UA1 and UA2 events showing missing energy-momentum associated with large $p_{1}$ photons or jets are sometimes taken as a first hint for the production of Susy particles. Obviously careful analyses with much more statistics are required. In $e^{+} e^{-}$collisions the limitation is essentially due to the available energy. SLC and LEP will be welcome. 
Experiments with missing energy-momentum are always difficult to interpret. Polarization can help for two reasons. Because of the difference in spin with respect to usual particles the production of Susy particles should present different polarization asymmetries. A good Susy signature is missing energy-momemtum associated to a special polarization asymmetry. Secondly polarization as usual will help for amplitude analysis and for the determination of the basic couplings of the new particles. This will be necessary in order to prove that these particles are the ones predicted by the supersymmetric gauge theory and in order to choose among the various possible models.

We show that a typical feature of Susy particle production is a change of $\mathrm{sign}$ of certain polarization asymmetries with respect to the case of ordinary particle production. This is a very profound property of supersymmetric invariance related to the so-called non-renormalization theorems /2,3/. Tests could easily be done at $e^{+} e^{-}$colliders.

II - TYPICAL ILLUSTRATIONS

A/ Gluino production in polarized $p\left(\frac{p}{p}\right.$ collisions

The subprocess $g g \rightarrow \tilde{g} g$ (as well as other gaugino processes) has been considered as one of the most promissing source of Susy particle in hadronic collisions / $/ 7,8 /$. The peculiar helicity dependence of this process as compared to that of the usual process $g g \rightarrow g g$ was noticed by Craigie et al $/ 9 /$. Neglecting $\mathrm{m}_{\tilde{g}}$ the cross-sections read :

$$
\begin{aligned}
& \frac{d \sigma}{d t}\left(g^{h} h^{\prime} \rightarrow g g\right)=\frac{9 c}{4 s^{2}}\left[\left(1-\frac{t u}{s^{2}}\right) \frac{t^{2}+u^{2}}{t u}\right]\left(1-h h^{\prime}\right) \\
& \frac{d \sigma}{d t}\left(g g^{h} h^{\prime} \rightarrow g g\right)=\frac{9 c}{2 s^{2}}\left[3-\frac{s u}{t^{2}}-\frac{s t}{u^{2}}-\frac{u t}{s^{2}}+\left(-3+\frac{2 s^{2}}{u t}+\frac{u t}{s^{2}}\right) h h^{\prime}\right]
\end{aligned}
$$

where $h, h^{\prime}$ are the initial gluon helicities. The corresponding asymmetries are shown in Fig. 2. The peculiar factor $\left(1-h^{\prime}\right)$, i.e. $A_{L L}=-1$ for gluino production

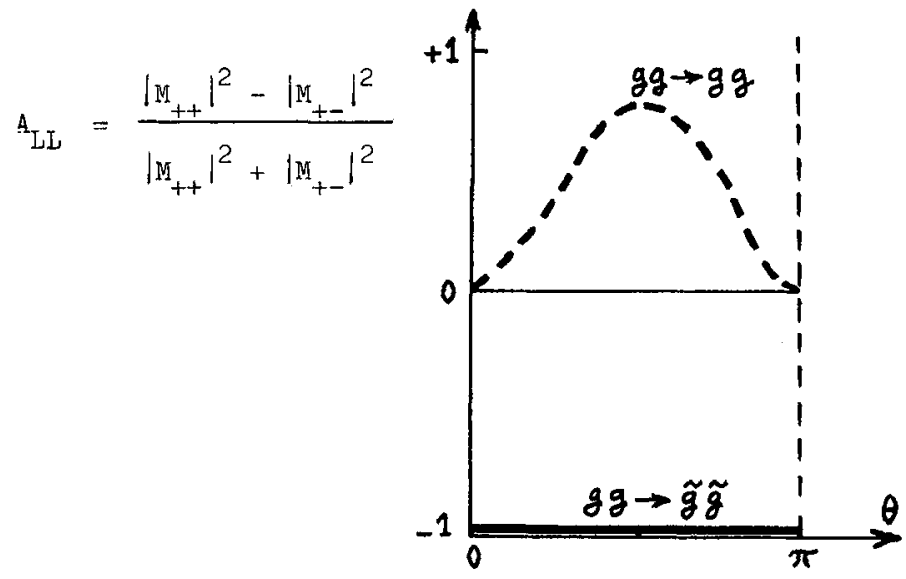

Fig. 2 : Helicity asymmetries in $g g \rightarrow g g$ and $g g \rightarrow g g / 9 /$. 
is typical of this subprocess (helicity conservation along the fermion line) and should not surprise those familiar with $e^{+} e^{-} \rightarrow y \gamma$ or $Y y \rightarrow q q / 10 /$. . The change of sign of $A_{L L}$ with respect to $g g \rightarrow g g$ survives gluino mass effects and convolution with spin-dependent parton distributions inside polarized $p$ and $(\vec{p})$ as shown by Craigie et al. These authors considered also the other subprocesses $\mathrm{gq} \rightarrow \tilde{\mathrm{gq}}$, gg $\rightarrow \tilde{\mathrm{qq}}$ and $\mathrm{qq} \rightarrow \tilde{\mathrm{qq}}$. Their conclusion is that missing-energy-momentum events due to Susy particle production should be clearly associated with helicity asymmetries in polarized $p^{(-)}$collisions.

B/ Linear polarization of direct photons produced in $p(-)$ collisions

This is an example of final polarization produced with unpolarized beams. The subprocesses $q+g \rightarrow q+\gamma$ and $q+\bar{q} \rightarrow g+\gamma$ are the standard sources of direct photons in hadronic collisions. QCD calculations even by including heavy quark mass effects $/ 11 /$ predict only a negligible photon polarization :

$$
P_{\gamma}=\frac{\left|M_{\perp}\right|^{2}-|M /|^{2}}{\left|M_{\perp}\right|^{2}+|M / /|^{2}} \lesssim 10^{-3}
$$

Antoniadis et al /11/ remarked that larger mass effects arise in supersymmetric calculations which use the subprocesses $\tilde{q}+g \rightarrow \tilde{q}+\gamma$ and $q \tilde{q}+\tilde{q}-\tilde{g}+\gamma$. The essentinl difference with the effect of massive top quarks in that the probability of finding squarks $\widetilde{q}$ and giuinos $\widetilde{g}$ inside proton structure is much larger. According to Kounnas and Ross /12/ for large $Q^{2}$ (deep structure) $32 \%$ of proton momemtum is carried by Susy partons. With such an input and $\underset{\sim}{\mathrm{q}}=20 \mathrm{GeV}, \mathrm{m}_{\mathfrak{g}} \lesssim 4 \mathrm{GeV}$, Antoniadis et al get a linear polarization approaching $10 \%$ for $\delta_{s}=540 \mathrm{GeV}$ and $p_{\perp}^{\gamma} \simeq 30 \mathrm{GeV}$. This is a good illustration of Susy effects. However the rate of large $p_{\perp}$ photons is small and a $10 \%$ linear polarization is not an easy measurement to do.

The Susy-parton contents of the proton could be tested in e-p collisions with HERA. This was studied by Jones and Llewellyn Smith/12/ but their conclusions were not very optimistic. The available energy for the subprocesses will be just sufficient and the identification of susy particles among the background will be difficult. For more details see the report by $P$. Schmuser at this symposium.

\section{C/ Scalar fermion production in $e^{+} e^{-}$collisions}

$J$. Soffer $/ 13 /$ remarked that the signs of the $\cos 2 \varphi$ dependence for transversally polarized $e^{ \pm}$beams differ in $e^{+} e^{-} \rightarrow \widetilde{f f}$ and in $e^{+} e^{-} \rightarrow f \vec{f}$.

Precisely through one photon annihilation one has :

$$
\begin{aligned}
\frac{d \sigma}{d \Omega}\left(e^{+} e^{-}-\tilde{f f}\right)= & \frac{\alpha^{2} Q_{f}^{2} \beta^{3}}{8 s} \sin ^{2} \theta\left(1-P_{\perp}^{+} P_{\perp}^{-} \cos 2 \varphi\right) \\
\frac{d \sigma}{d \Omega}\left(e^{+} e^{-} \rightarrow f f\right)= & \frac{\alpha^{2} Q_{f}^{2} \beta}{4 s}\left[2-\beta^{2} \sin ^{2} \theta+\beta^{2} \sin ^{2} \theta P_{\perp}^{+} P_{\perp}^{-} \cos 2 \varphi\right] .
\end{aligned}
$$

These expressions are exactly those of $e^{+} e^{-} \rightarrow \pi^{+} \pi^{-}$and $e^{+} e^{-} \rightarrow p \bar{p}$ in the pointlike limit $/ 10 /$. The reason of this change of sign is very general (compare $|\vec{\varepsilon} \cdot \vec{p}|^{2}$ to $|\vec{\varepsilon} \cdot(\vec{\sigma} \times \vec{p})|^{2}$ ), independent of signs of coupling constants and 
remains valid when one includes the $z^{\circ} / 14 /$.

This should give a clear way of identifying scalar particle production in $\mathrm{e}^{+} \mathrm{e}^{-}$ annihilation hidden in a background of spin $\frac{1}{2}$ lepton or hadron production.

\section{III - SUSY PARTICLE PRODUCTION IN POLARIZED $\mathrm{e}^{+} \mathrm{e}^{-}$COLLISIONS}

The preceding example motivated a more general study of the polarization properties in Susy particle production. I now report on a work in progress by P. Chiappetta, $J$. Soffer, P. Sorba, P. Taxil and myself $/ 14 /$. We consider both longitudinal ( $/ /$ ) and transverse $\left(P_{1}\right) e^{ \pm}$beam polarizations. We treat several types of susy particles (sfermions and gauginos) and include the decay distribution effects. Cross-section in $e^{+} e^{-}$collisions have the general helicity structure /10/ :

$$
\begin{aligned}
\frac{\mathrm{d} \sigma}{\mathrm{d} \Omega}= & \left(1-\mathrm{P}_{/}^{-} \mathrm{P}^{+} / /\right) \mathrm{X}_{1}+\left(1+\mathrm{P}^{-} / \mathrm{P}^{+} / /\right) \mathrm{X}_{1}^{\prime}+\left(\mathrm{P}_{/}^{+}-\mathrm{P}^{-} / /\right) \mathrm{X}_{2}+\left(\mathrm{P}_{/}^{+}+\mathrm{P}^{-} / /\right) \mathrm{X}_{2}^{\prime} \\
& +\left(\mathrm{P}_{1}^{-}-\mathrm{P}_{1}^{+}\right)\left[\mathrm{X}_{6} \cos \varphi+\mathrm{X}_{7} \sin \varphi\right]+\left(\mathrm{P}_{1}^{-}+\mathrm{P}_{\perp}^{+}\right)\left[\mathrm{X}_{6}^{\prime} \cos \varphi+\mathrm{X}_{7}^{\prime} \sin \varphi\right] \\
& +\mathrm{P}_{1}^{+} \mathrm{P}_{\perp}^{-}\left[\mathrm{X}_{3} \cos 2 \varphi+\mathrm{X}_{4} \sin 2 \varphi+\mathrm{X}_{5}\right] .
\end{aligned}
$$

We discuss the following longitudinal and transverse asymmetries :

and

$$
A_{\perp}^{\circ}=\left(\frac{\sigma^{\uparrow \downarrow}-\sigma^{\uparrow}}{\sigma^{\uparrow \downarrow}+\sigma^{\uparrow \uparrow}}\right)_{\varphi=0}=\frac{X_{3}+X_{5}}{X_{1}+X_{1}^{\prime}}
$$

$$
\mathrm{A} / /=\frac{\sigma^{+}-\sigma^{-}}{\sigma^{+}+\sigma^{-}}=\frac{X_{2}^{\prime}-X_{2}}{X_{1}+X_{1}^{\prime}}
$$

a) $e^{+} e^{-} \rightarrow \widetilde{\mu}_{L} \widetilde{\widetilde{\mu}}_{L}, \widetilde{\mu}_{\mathrm{R}} \overline{\widetilde{\mu}}_{\mathrm{R}}$ and decay $\tilde{\mu}_{\mu} \rightarrow \tilde{\gamma}$.

We compute the cross-section due to annihilation through $\gamma$ and $Z^{\circ} / 14 /$.

It contributes to $x_{i}(i=1,2,3,4)$. Fig. 3a shows the longitudinal asymmetry which mainly results from the vector-axial combination of the $\gamma-z^{\circ}$ interference and which obviously changes sion from $\tilde{\mu}_{L}$ to $\tilde{\mu}_{R}$. If $\tilde{\mu}_{L}$ and $\tilde{\mu}_{R}$ are not degenerate one here has a very good signal for scalar lepton production. If $\tilde{\mu}_{\mathrm{L}}$ and $\tilde{\mu}_{\mathrm{R}}$ are degenerate the sum of their contributions to $\mathrm{A} / /$ exactly coincides with the one of $e^{+} e^{-} \rightarrow \mu^{+} \mu^{-}$.

Fig. 3b shows the resulting transverse asymmetry in the case of $\mathrm{m}_{\widetilde{\mu}_{\mathbf{H}}}=20$ and $40 \mathrm{GeV}$. As expected it changes sign with respect to the case of $e^{+} e^{-} \rightarrow \mu^{+} \mu^{-}$. Even if one could not distinguish $e^{+} e^{-} \rightarrow \mu^{+} \mu^{-}$from $e^{+} e^{-} \rightarrow \mu^{+} \mu^{-} \tilde{\gamma \gamma}$ the averaged transverse asymmetry is still very different from the pure $\mu^{+} \mu^{-}$case as soon as we stand above the $Z^{\circ}$ peak. For example at $\sigma_{S}=100 \mathrm{GeV}$ for $m_{\widetilde{\mu}}=20 \mathrm{GeV}, A_{\perp}^{0}$ is equal to +0.40 , the pure $\mu^{+} \mu^{-}$case is -0.33 and the average is -0.16 . 


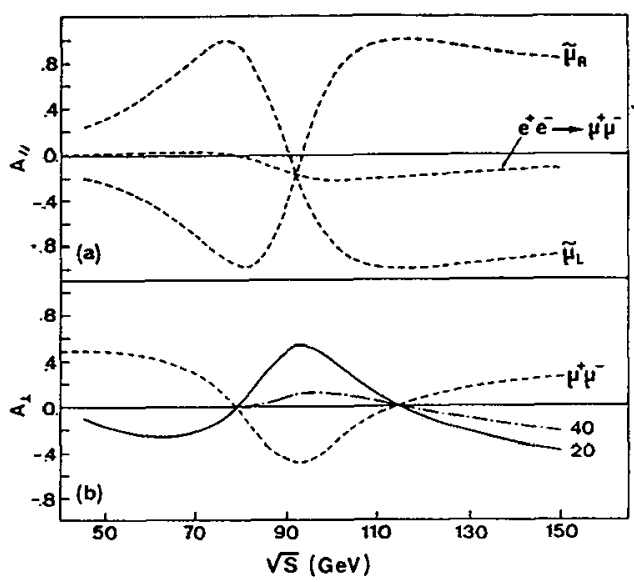

Fig. 3 : Longitudinal and Transverse asymmetries in $e^{+} e^{-} \rightarrow \widetilde{\mu_{\mu}} \rightarrow \mu^{+} \mu^{-} \widetilde{\gamma \gamma} / 14 /$. b) $e^{+} e^{-}-\tilde{e} \bar{e}$ and decay $\tilde{e} \rightarrow e+\tilde{y}$.

In addition to annihilation through $\gamma$ and $z^{\circ}$ which produce $\tilde{l}_{L_{L}} \overline{\widetilde{l}}_{\text {and }} \widetilde{x}_{R} \bar{x}_{R}$ we also have gaugino $(\widetilde{\gamma}, \widetilde{z}, \ldots)$ exchanges which contribute $/ 15 /$ to the 4 processes $e^{+} e^{-} \rightarrow \widetilde{l}_{L} \overline{\vec{l}}_{L}, \widetilde{x}_{R} \overline{\mathbb{l}}_{R}, \widetilde{l}_{L} \bar{l}_{R}, \widetilde{l}_{R} \overline{\vec{l}}_{L}$ and gives new $x_{1,2}^{\prime}$ terms. Similarly to the case of photon exchange in Bhabha scattering the gaugino exchange processes increase the rates, peak the angular distributions but do not directly contribute to the transverse asymmetry (except by their interference with the annihilation terms).

Around the $Z$ peak where the annihilation terms dominate the gross features of the asymmetries are similar to those of $e^{+} e^{-} \rightarrow \sim_{\mu}^{+} \tilde{H}^{-}$. See for example the transverse asymmetry in Fig. 4 compared to the one of $e^{+} e^{-} \rightarrow e^{+} e^{-}$. For $\sqrt{s}=100 \mathrm{GeV}$ the difference with Bhabha scattering is even very strong for the longitudinal asymmetry especially in the backward region as shown in Fig. 5.

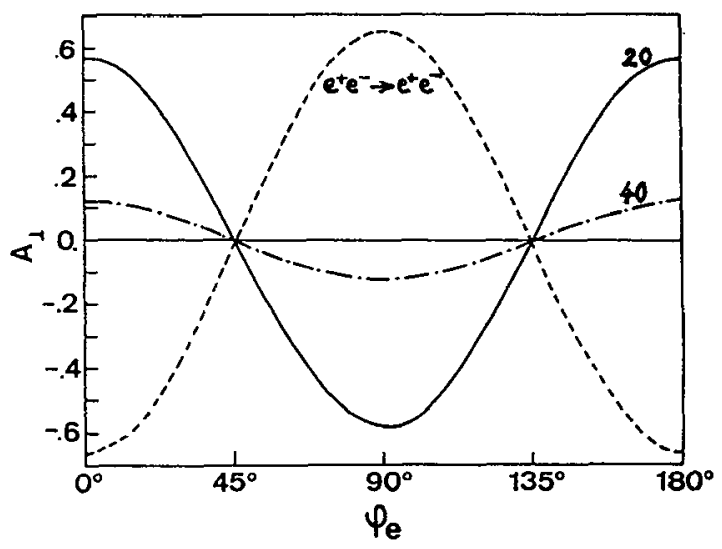

Fig. 4 : Transverse asymmetry in $e^{+} e^{-} \rightarrow \tilde{e} \bar{e}-e^{+} e^{-} \gamma \gamma$, integrated on $\theta_{e}$ between 900 and 1400 at $\sqrt{s}=m_{Z} / 14 /$. 


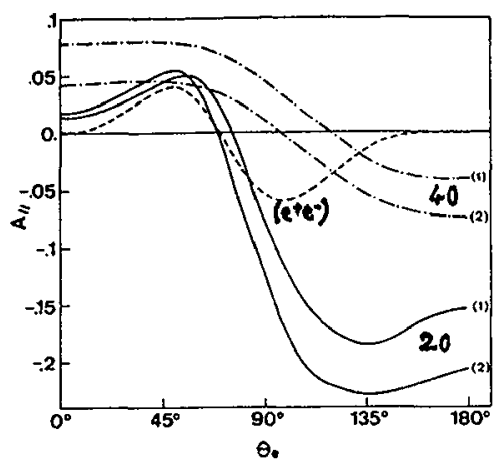

Fig. 5 : Longitudinal asymmetry in $e^{+} e^{-} \rightarrow \tilde{e} \bar{e} \rightarrow e^{+} e^{-} \tilde{Y Y}$ at $\sqrt{s}=100 \mathrm{GeV} / 14 /$.

Even if one could not experimentally distinguish $e^{+} e^{-} \rightarrow e^{+} e^{-}$from $e^{+} e^{-} \rightarrow e^{+} e^{-} \tilde{Y \gamma}$ the resulting asymmetries are still different from the pure Bhabha case. For example at $\sqrt{s}=m_{Z}$ the pure Bhabha transverse asymmetry is $A_{1}^{0}=-0.66$, the pure $\tilde{\mathrm{e}} \overline{\mathrm{e}}$ one is +0.58 and the averaged one is -0.27 .

\section{c) other processes.}

Applications to $e^{+} e^{-}-\overrightarrow{\tilde{v}_{v}}, \widetilde{\bar{q}}$ are straighforward. Other interesting channels are $e^{+} e^{-} \rightarrow 2$ gauginos or higgsinos $\left(\tilde{Y}, \widetilde{Z}, \widetilde{W}^{ \pm}, \widetilde{H}^{ \pm}, \ldots\right)$. We suspect that the polarization effect on $e^{+} e^{-} \rightarrow \widetilde{\gamma Y}$ will be notably different from the one on its background $e^{+} e^{-} \rightarrow \gamma \bar{\nu}$ so that much better limits on $\mathbb{m}_{\widetilde{e}}$ and $\underset{\widetilde{\gamma}}{\mathfrak{m}}$ could already be given with polarized $e^{ \pm}$beams. These calculations are in progress $/ 14 /$.

\section{IV - CONCLUDING REMARKS}

In the various examples that we have treated thestriking feature of susy particle production in polarized collisions is the change of sign of certain polarization asymmetries. This is not an accident but a very deep property of Supersymmetry. This change of sign between fermion and boson contributions is the basic ingredient of the so-called non renormalization theorem /2,3/. For example in $e^{+} e^{-}$collisions one can explicitely and very easily check (adding $e^{+} e^{-} \rightarrow f \bar{f}, \widetilde{f}_{L_{L}} \overline{\bar{f}_{L}}$ and $\widetilde{f}_{R} \widetilde{\widetilde{f}}_{R}$ ) that when summing over a complete susymultiplet and neglecting the mass differences the total transverse asymmetry (i.e. $X_{3}$ term of $\frac{d \sigma}{d \Omega}$ ) exactly cancels. When one remembers that $e^{+} e^{-}$annihilation cross-section is related to the renormalization factor of boson propagators this may not be a surprise. Polarized collisions will not only be of help for detecting Susy particles, it will be a way of testing one of the deepest property of Supersymmetric invariance.

Azimuthal $\cos 2 \varphi$ dependence of the cross-section for naturally polarized $e^{ \pm}$ beams was already observed at various $e^{+} e^{-}$colliders. I especially remember the 1975 experiment /16/ at Spear which clearly showed that quarks have spin $\frac{1}{2}$.

Why not a similar experiment at higher energies revealing Supersymmetry ? 


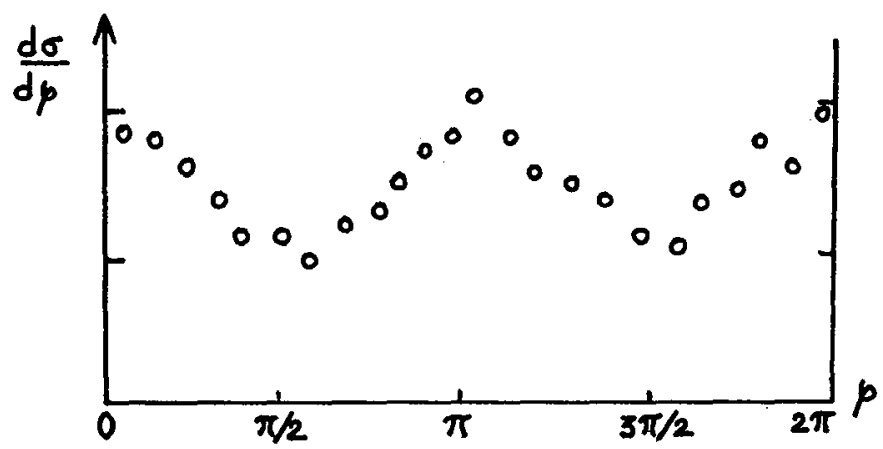

Fig. 6 : Azimuthal dependence of $\sigma\left(\mathrm{e}^{+} \mathrm{e}^{-} \rightarrow\right.$ hadrons $)$ measured by SLAC-LBL at Spear $(\sqrt{s}=7.4 \mathrm{GeV})$ in $1975 / 16 /$.

\section{REFERENCES}

[1] GOL'FAND Y.A. and LIKHTMAN E.P., JETP lett. 13 (1971) 323. VOLKOV D. and AKULOV V.P., Phys. Lett. 46B (1973) 109. WESS J. and ZUMINO B., Nucl. Phys.. B70 (1974) 39.

[2j FAYET P. and FERRARA S., Phys. Rep. 32C (1977) 249. VAN NIEUWENHUIZEN P., Phys. Rep. 68C (1981) 189.

NILLES H.P., preprint UGVA-DPT 1983/12-412, to appear in Phys. Rep.

[3] FAYET P., Proc. XXI $I^{\text {st }}$ Int. Conf. High Energy Physics, Paris, Jour. Phys. C3 (1982) 673.

ELIIS J., Proc. Int. Symp. Lepton and Photon Int. at High Energies, Cornell, 1983, p. 439.

CERN Workshop on Supersymmetry, Phys. Rep. 105C (1984) 1.

[4] ELIIS J., Lect, at Adv. Study Inst. Munich (1983), TH. 3802-CERN.

FAYET P., Lect at 3 rd Trieste School (1984), LPTENS 84/17.

KOUNNAS C., Talk at $\overline{4}^{\text {th }}$ Top. Workshop p p Bern (1984), LPTENS 84/15.

[5] YAMADA S., Proc. Int. Symp. Lepton and Photon Int. at High Energies, Cornell, 1983 , p. 525.

[6] BAND H.R., Talk at XIX Renc. Moriond, La Plagne 1984, SLAC-PUB-3335.

[7] HABER H.E. and KANE G.L., Phys. Lett. 142B (1984) 212. ELLIS J. and KOWALSKI H., Phys. Lett. $\overline{142 B}(1984) 441$. ALTARELLI G., MELE B. and PETRARCA S., TH. 3822 - CERN. BARGER V. et al, Phys. Rev. Lett 23 (1984) 641.

[8] KANE G.L. and LEVEILIE J.P., Phys. Lett. 112B (1982) 227. HARRISON P.R. and ILEWELLYN SMITH C.H., Nucl. Phys. B213 (1983) 223.

[9] CRAIGIE N.S., HIDAKA K. and RATCLIFFE P., Phys. Lett. 129B (1983) 310.

[10] RENARD F.M., Basics of $e^{+} e^{-}$collisions, ed. Frontière (1981).

[11] ANTONIADIS I., CONTOGOURIS A.P. and TANAKA H., Phys. Rev. D29 (1984) 1362.

[12] KOUNNAS C, and ROSS D., Nucl. Phys. B214 (1983) 317. JONES S.K. and LLEWELLYN SMITH C.H., Nucl. Phys. B217 (1983) 145.

[13] SOFFER J., Talk at Physics in Collisions, Como 1983, ed. Frontières, p. 135.

[14] CHIAPPETTA P., RENARD F.M., SOFFER J., SORBA P. and TAXIL P., in preparation.

[15] GLÜCK M. and REYA E., Phys. Lett. 130B (1983) 423.

HIDAKA K., KOMATSU $H$. and RATCLIFFE P., preprint 1984.

[16] SCHWITTERS R.F. et al, Phys. Rev. Lett. 25 (1975) 1320. 